\title{
Stability of slender beams and frames resting on 2D elastic half-space
}

Received: 6 February 2012 / Accepted: 31 July 2012 / Published online: 15 August 2012

(c) Springer-Verlag 2012

\begin{abstract}
Making use of a mixed variational formulation based on the Green function of the substrate, which assumes as independent fields the structure displacements and the contact pressure, a simple and efficient finite element-boundary integral equation coupling method is derived and applied to the stability analysis of beams and frames resting on an elastic half-plane. Slender Euler-Bernoulli beams with different combinations of end constraints are considered. The examples illustrate the convergence to the existing exact solutions and provide new estimates of the buckling loads for different boundary conditions. Finally, nonlinear incremental analyses of rectangular pipes with compressed columns and free or pinned foundation ends are performed, showing that pipes stiffer than the soil may exhibit snap-through instability.
\end{abstract}

Keywords Buckling · Euler-Bernoulli beam · Soil-structure interaction · Frictionless contact · Mixed finite elements · Flamant solution

\section{Introduction}

The stability of beams and frames resting on a substrate or soil is important in many engineering fields and has been previously studied by many researchers. In the civil engineering field, examples of this problem are the stability of road pavements and the lateral buckling of welded railway rails. In this context, the pioneering works of Wieghardt [1] and Prager [2] are based on the assumption that the beam is resting on a continuously distributed set of springs. In 1937, Biot [3] studied the problem of an infinite beam on an elastic continuum loaded by vertical forces and, in the same year, Reissner [4] first studied the related stability problem. Then, the interest in this problem grew, motivated by early structural problems of sandwich elements, and Gough et al. [5] extended Biot and Reissner results including various conditions of contact between the infinite beam and the elastic half-space. The study of sandwich elements continued up to recent years [6-8]. Recently, the main interest has been motivated by thin film buckling and the research has been driven by developments in electronics industry $[9,10]$. In this context, the case of buckling without delamination is often called wrinkling.

In [11], the buckling of a simply supported beam on Winkler soil is studied. Other boundary conditions, such as beam with clamped ends and beam with free ends, were studied and compared with the former [12]. In

\author{
N. Tullini $(\bowtie) \cdot$ A. Tralli $\cdot$ D. Baraldi \\ Department of Engineering, \\ University of Ferrara, Ferrara, Italy \\ E-mail: nerio.tullini@unife.it \\ A. Tralli \\ E-mail: tra@unife.it \\ D. Baraldi \\ E-mail: daniele.baraldi@unife.it
}


the context of sandwich plates, Goodier and Hsu [13] underlined the presence of nonsinusoidal local buckling modes located at the beam ends. Assuming the more realistic relationship between foundation pressure and beam displacement suggested in [1], Smith [14] determined the buckling loads of a beam with pinned ends. With regard to the simply supported beam of finite length on an elastic half-plane, Gallagher [15] evaluated the buckling loads using a governing fourth-order integro-differential equation solved by means of a series of Chebyshev polynomials, whereas Bosakov [16] applied the Ritz method, but its solution is accurate for short beams stiffer than the soil only. To the authors' knowledge, no more analytical or numerical analyses concerning the buckling of a slender beam of finite length in frictionless contact with an elastic half-plane are available.

In the present work, the critical loads of Euler-Bernoulli beams with finite length resting in frictionless contact with an elastic two-dimensional half-space are evaluated by generalizing the finite element-boundary integral equation (FE-BIE) coupling method proposed in [17], where the static analysis of foundation beams with free ends is considered. In particular, the soil is represented as an elastic half-space in plane state conditions. Hence, making use of the Green function of the soil, a proper relationship between beam deflection and contact pressure is adopted. A mixed variational formulation is used, with variational functions represented by structure displacements and pressure soil reaction. The corresponding FE model for the soil-beam system adopts classical beam elements and constant soil reaction underlying each foundation element. Unlike what is claimed in [17], possible constraints cannot be applied to global stiffness and geometric matrices as usual because the Green function of the soil holds for a half-plane loaded by a point force normal to its boundary, which must be free to deform elsewhere. In this paper, a penalty approach is adopted to include constraint equations in the mixed variational formulation of the foundation-soil system. Making use of a parameter that takes into account both the beam slenderness and the soil stiffness, comparisons with analytical solutions and traditional two-dimensional (2D) FEs are given.

Moreover, rectangular frames on an elastic half-space with compressed columns are considered. Buckling loads and mode shapes are determined varying the soil stiffness for two different restraint conditions. In addition, the geometric nonlinear behavior of the frames is investigated and the load multipliers at the limit point are compared with the buckling loads, showing that pipes stiffer than the soil may exhibit snap-through instability. It is worth noting that conventional 2D FEs model describes hardly the global buckling modes of frames on an elastic half-space because many local modes characterize the substrate near the column ends.

\section{Basic relationships}

An elastic beam of length $L$, cross-section height $h$, and width $b$, resting on a semi-infinite linearly elastic substrate, is referred to a Cartesian coordinate system $(0 ; x, y)$, where $x$ coincides with both the centroidal axis and the boundary of the half-plane and $y$ is directed downward. In the following, $E_{b}$ and $E_{s}$ indicate the Young moduli of beam and substrate, respectively. Analogously, Poisson ratios of beam and substrate are denoted by $v_{b}$ and $v_{s}$, respectively. Generalized plane stress or plane strain regime is considered; in the latter case, both the beam and the half-plane have an unitary values of the width $b$. The beam is loaded at the ends by a concentrated compressive force $P$ as shown in Fig. 1. A vertical load $p(x)$ distributed along the beam axis $x$ can also be considered. In the interface between beam and soil, frictionless and bilateral conditions are assumed. Consequently, a vertical soil reaction $r(x)$ is enforced to both beam and substrate and the vertical displacement $v(x)$ of the beam coincides with that of the half-plane boundary.

The potential energy $\Pi_{b}$ of the beam, including second-order effects, can be written as [18]:

$$
\Pi_{b}=\frac{1}{2} \int_{L}\left[D_{b}\left(v^{\prime \prime}(x)\right)^{2}-P\left(v^{\prime}(x)\right)^{2}\right] \mathrm{d} x-b \int_{L}(p(x)-r(x)) v(x) \mathrm{d} x
$$

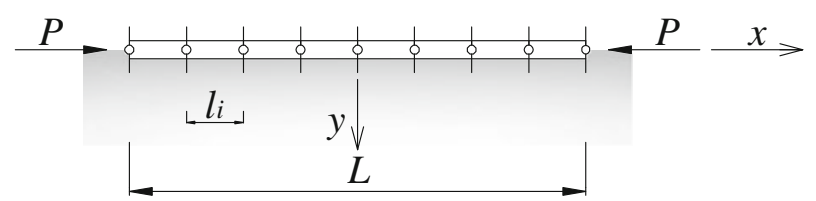

Fig. 1 Foundation beam with axial load subdivided into equal FEs 
where prime denotes differentiation with respect to $x$ and $D_{b}=E_{0} b h^{3} / 12$ is the bending rigidity, with $E_{0}=E_{b}$ or $E_{0}=E_{b} /\left(1-v_{b}^{2}\right)$ for a generalized plane stress or plane strain state, respectively.

The potential energy of the soil is given by [17]:

$$
\Pi_{s}=-\frac{b}{2} \int_{L} r(x) v(x) \mathrm{d} x=-\frac{b}{2} \int_{L} r(x) \mathrm{d} x \int_{L} g(x, \hat{x}) r(\hat{x}) \mathrm{d} \hat{x}
$$

where the vertical displacement $v(x)$ is replaced by the boundary integral equation known as Flamant solution [19], which uses the Green function $g(x, \hat{x})$ corresponding to the solution to the elastic problem for a homogeneous isotropic half-plane loaded by a point force normal to its boundary:

$$
g(x, \hat{x})=-\frac{2}{\pi E} \ln |x-\hat{x}|
$$

with $E=E_{s}$ or $E=E_{S} /\left(1-v_{s}^{2}\right)$ for a generalized plane stress or plane strain state, respectively.

Many constraint equations $R_{i}\left(v, v^{\prime}\right)=0$ between displacements or rotations may be assigned along the beam axis. For example, a pinned-pinned beam requires the equation $v(L / 2)-v(-L / 2)=0$. These constraint equations can be included in the total potential energy $\Pi$ of the beam-substrate system by means of a penalty approach $[20]$ :

$$
\Pi(v, r)=\Pi_{b}(v, r)+\Pi_{s}(r)+\frac{k}{2} \sum_{i}\left[R_{i}\left(v, v^{\prime}\right)\right]^{2}
$$

where $k$ is the penalty parameter, whose value should be large enough to satisfy the constraint equations accurately. For beams with free ends, the rigid body displacement related to the Flamant solution can be removed by choosing an arbitrary abscissa $\bar{x}$ where a null value of $v(\bar{x})$ is forced. It is worth noting that Flamant solution (3) holds for a half-plane loaded by a point force normal to its boundary, which must be free to deform elsewhere. The penalty approach allows to reformulate a problem with constraints as one without constraints. This topic was not detailed in [17].

A simple discretization of the beam-substrate system can be created by subdividing the beam into FEs of length $l_{i}$ (Fig. 1) and considering a constant soil reaction for each foundation element [17]. With regard to the beam shape functions, classical Hermitian polynomials are assumed [20]. For a prismatic beam, the stationarity condition of the total potential energy $\Pi$ written in discrete form provides the following system:

$$
\left[\begin{array}{cc}
\frac{D_{b}}{L^{3}}\left(\widetilde{\mathbf{K}}_{b}-\frac{P L^{2}}{D_{b}} \widetilde{\mathbf{K}}_{g}\right) & b \mathbf{H} \\
b \mathbf{H}^{\mathrm{T}} & -\frac{b}{E} \widetilde{\mathbf{G}}
\end{array}\right]\left\{\begin{array}{c}
\mathbf{q} \\
\mathbf{r}
\end{array}\right\}=\left\{\begin{array}{c}
b \mathbf{F} \\
\mathbf{0}
\end{array}\right\}
$$

where the vector $\mathbf{q}$ collects the nodal displacements, $\mathbf{r}$ denotes the vector of the constant soil reactions, $\mathbf{F}$ is the vector of the external loads, $D_{b} / L^{3} \widetilde{\mathbf{K}}_{b}$ is the elastic stiffness matrix of the beam, $P / L \widetilde{\mathbf{K}}_{g}$ is the geometric (or incremental) stiffness matrix, and the elements of the matrices $\mathbf{H}$ and $\widetilde{\mathbf{G}}$ are reported in "Appendix", together with the element matrices $\widetilde{\mathbf{K}}_{b i}$ and $\widetilde{\mathbf{K}}_{g i}$. The system in Eq. (5) yields the following solution:

$$
\begin{aligned}
& \mathbf{r}=E \widetilde{\mathbf{G}}^{-1} \mathbf{H}^{\mathrm{T}} \mathbf{q} \\
& {\left[\widetilde{\mathbf{K}}_{b}-\lambda \widetilde{\mathbf{K}}_{g}+\widetilde{\mathbf{K}}_{\text {soil }}\right] \mathbf{q}=\mathbf{F} \frac{b L^{3}}{D_{b}}}
\end{aligned}
$$

where the stiffness matrix of the soil

$$
\widetilde{\mathbf{K}}_{\text {soil }}=(\alpha L)^{3} \mathbf{H} \widetilde{\mathbf{G}}^{-1} \mathbf{H}^{\mathrm{T}},
$$

the load multiplier $\lambda$ and the parameter $\alpha L$ are defined as follows;

$$
\begin{array}{r}
\lambda=\frac{P L^{2}}{D_{b}}, \\
\alpha L=\sqrt[3]{\frac{E b L^{3}}{D_{b}}} .
\end{array}
$$


According to references [3,17,21], the parameter $\alpha L$ given by Eq. (9b) describes the beam-substrate system. Low values of $\alpha L$ characterize short beams stiffer than the soil, whereas high values of $\alpha L$ describe more flexible beams, this latter case is suitable to represent long beams on a stiff soil.

The adopted mixed finite element is particularly simple and effective, as shown in [17] for the static case, where the numerical properties of the proposed FE model are also discussed. With regard to the determination of critical load $P_{\mathrm{cr}}$, a homogeneous system associated with Eq. (7) must be considered and the buckling loads are given by the roots $\lambda_{\text {cr }}$ of the equation det $\left[\widetilde{\mathbf{K}}_{b}-\lambda \widetilde{\mathbf{K}}_{g}+\widetilde{\mathbf{K}}_{\text {soil }}\right]=0$, which can be suitably reduced to a standard eigenvalue problem. Making use of Eq. (9a) and the definition of Euler critical load:

$$
P_{\mathrm{cr}, E}=\frac{\pi^{2} D_{b}}{L^{2}}
$$

the dimensionless buckling loads turn out to be given by $P_{\mathrm{cr}} / P_{\mathrm{cr}, E}=\lambda_{\mathrm{cr}} / \pi^{2}$.

In the case of a structure connected to the foundation beam, system (7) can be partitioned as shown in [17], where nodal displacements with or without nodes shared with the foundation beam are selected. Moreover, the geometric matrix of each beam FE is handled in the usual way.

\section{Slender beams with different boundary conditions}

\subsection{Convergence test for beams with free ends}

In order to compare the proposed model with a classical model that uses $2 \mathrm{D}$ elastic elements to describe the soil, a convergence test is presented first. Two different cases, representing a quite stiff beam $(\alpha L=5)$ and a flexible beam $(\alpha L=25)$, are considered. In both cases, the foundation beam is modeled as an Euler-Bernoulli beam subdivided into equal FEs, with a number of elements $n_{\mathrm{el}}$ equal to powers of two up to $256 \mathrm{FEs}$. Each beam element of the proposed model includes a single soil element, whereas in the 2D model, the soil is modeled by a square mesh of quadrilateral elements in plane state. The mesh used for the soil has a total width equal to $8 \mathrm{~L}$. At the boundaries, the displacements in the normal direction are fixed. Two nested square meshes, with width equal to $4 L$ and $2 L$, are built close to the foundation beam. Each edge of the quadrilateral elements of the smaller mesh has the same size of the beam elements. In Fig. 2, the case of the foundation beam subdivided into 4 beam FEs is shown. The adopted mesh for the 2D model allows to accurately determine the displacements underlying the foundation beam, with a number of elements lower than that required by a simple mesh of quadrilateral elements. The frictionless connection between beam and soil nodes is established by vertical master-slave links. The adopted 2D code uses a geometric stiffness matrix also for the 2D soil FEs.

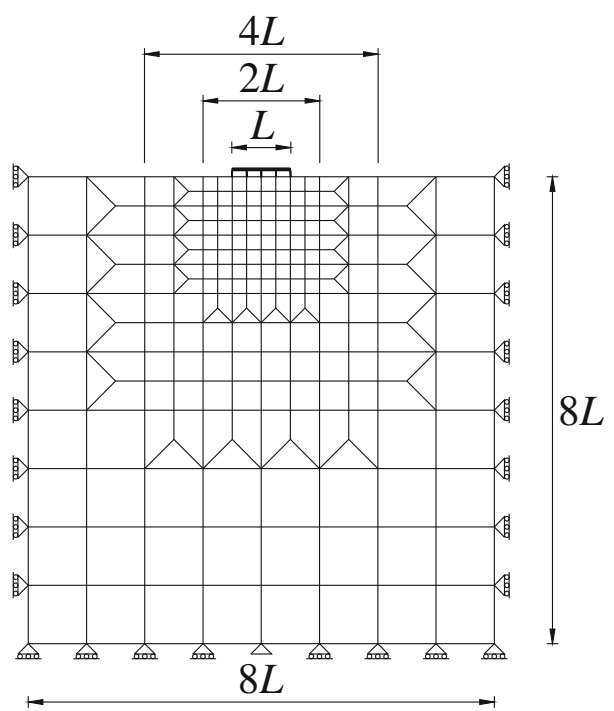

Fig. 2 Mesh adopted for the 2D model with foundation beam subdivided into 4 equal FEs 
Table 1 First three dimensionless critical loads $P_{\mathrm{cr}} / P_{\mathrm{cr}, E}$ for a beam with free ends corresponding to the present analysis (PA) or $2 \mathrm{D}$ models as a function of $n_{\mathrm{el}}$ for $\alpha L$ equal to 5 and 25

\begin{tabular}{|c|c|c|c|c|c|c|c|c|c|c|c|c|}
\hline \multirow[t]{3}{*}{$n_{\mathrm{el}}$} & \multicolumn{6}{|c|}{$\alpha L=5$} & \multicolumn{6}{|c|}{$\alpha L=25$} \\
\hline & \multicolumn{3}{|l|}{ PA } & \multicolumn{3}{|l|}{$2 \mathrm{D}$} & \multicolumn{3}{|c|}{ PA } & \multicolumn{3}{|l|}{$2 \mathrm{D}$} \\
\hline & 1st & 2nd & 3rd & 1st & 2nd & 3rd & 1st & 2nd & 3rd & 1st & 2nd & 3rd \\
\hline$\overline{2^{2}}$ & 1.688 & 1.889 & 4.956 & 2.267 & 2.939 & 4.917 & 6.776 & 9.240 & 29.47 & 19.45 & 25.48 & $\overline{46.13}$ \\
\hline $2^{3}$ & 1.880 & 2.131 & 5.008 & 2.159 & 2.630 & 4.993 & 23.81 & 23.85 & 65.54 & 60.80 & 62.31 & 65.65 \\
\hline $2^{4}$ & 1.949 & 2.233 & 5.019 & 2.087 & 2.474 & 5.017 & 40.58 & 40.65 & 77.51 & 61.61 & 61.69 & 75.05 \\
\hline $2^{5}$ & 1.977 & 2.279 & 5.022 & 2.046 & 2.396 & 5.022 & 47.60 & 47.66 & 78.08 & 57.85 & 57.89 & 77.36 \\
\hline $2^{6}$ & 1.990 & 2.300 & 5.023 & 2.025 & 2.356 & 5.022 & 50.14 & 50.20 & 78.16 & 55.21 & 55.26 & 77.97 \\
\hline $2^{7}$ & 1.996 & 2.311 & 5.023 & 2.014 & 2.337 & 5.022 & 51.19 & 51.25 & 78.17 & 53.71 & 53.77 & 78.12 \\
\hline $2^{8}$ & 1.999 & 2.316 & 5.023 & 2.008 & 2.327 & 5.022 & 51.66 & 51.72 & 78.17 & 52.93 & 52.98 & 78.16 \\
\hline $2^{11}$ & 2.004 & 2.318 & 5.023 & - & - & - & 52.06 & 52.11 & 78.17 & - & - & - \\
\hline
\end{tabular}
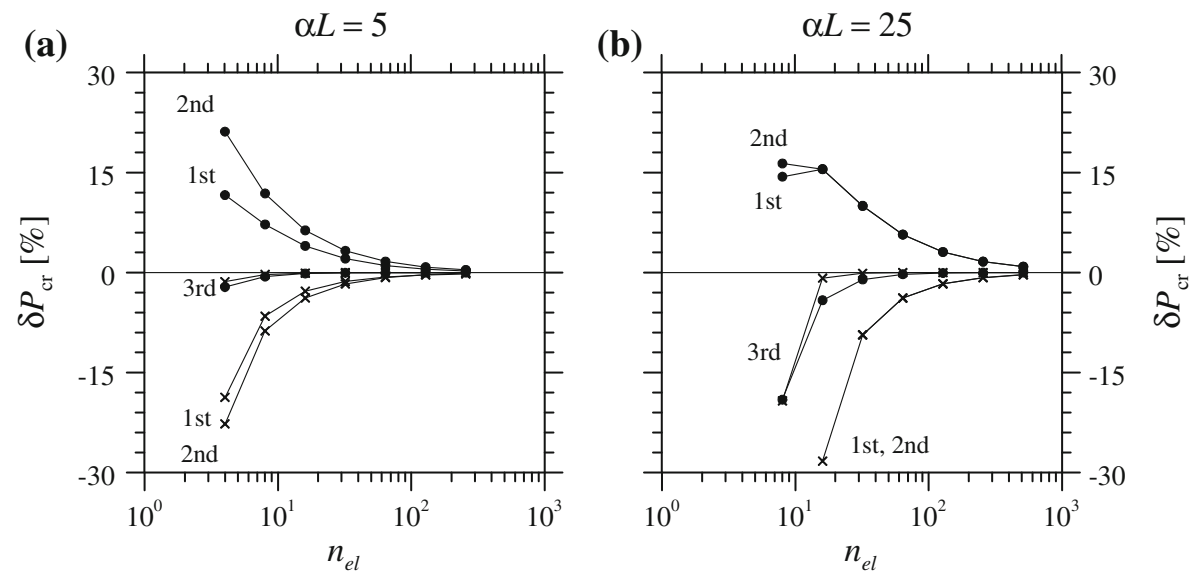

Fig. 3 Relative errors $\delta P_{\text {cr }}$ for first three buckling loads as a function of $n_{\mathrm{el}}$ for $\alpha L=5$ (a) and $\alpha L=25$ (b). Lines with crosses or dots correspond to present analysis or $2 \mathrm{D}$ models, respectively

The number of equations of the $2 \mathrm{D}$ model $n_{\mathrm{eq}}^{2 \mathrm{D}}$ is related to the number of equations of the present analysis $n_{\mathrm{eq}}^{\mathrm{PA}}$ by means of the following relation:

$$
n_{\mathrm{eq}}^{2 \mathrm{D}} \cong 2\left(n_{\mathrm{eq}}^{\mathrm{PA}}\right)^{2}
$$

$n_{\mathrm{eq}}^{\mathrm{PA}}=2 n_{\mathrm{el}}+2$ as usual.

The present analysis performed with a beam having 2,048 FEs is used as reference to determine the first three buckling loads $P_{\mathrm{cr}}^{\text {ref }}$, see Table 1 for $n_{\mathrm{el}}=2^{11}$. Moreover, Table 1 shows the first three dimensionless critical loads $P_{\mathrm{cr}} / P_{\mathrm{cr}, E}$, evaluated with the 2D model and the present analysis, as a function of $n_{\mathrm{el}}$ for $\alpha L$ equal to 5 and 25. Figure 3a, b shows the relative error $\delta P_{\mathrm{cr}}=\left(P_{\mathrm{cr}}^{\mathrm{FEM}}-P_{\mathrm{cr}}^{\mathrm{ref}}\right) / P_{\mathrm{cr}}^{\mathrm{ref}}$ as a function of $n_{\mathrm{el}}$ for $\alpha L=5$ and 25, respectively. Both methods converge with a rate lower than $n_{\mathrm{el}}^{-1}$ for the first two eigenvalues, but CPU time $t^{2 \mathrm{D}}$ of the 2D model is greater than $5\left(^{\mathrm{PA}}\right)^{2.5}$, where $t^{\mathrm{PA}}$ is the CPU time of the present analysis. Moreover, the relative errors are lower than $1 \%$ with at least 128 beam FEs. Thus, the present model can be considered effective for the determination of buckling loads and mode shapes, and a number of 256 equal FEs are adopted for all cases reported in this section. In the following, beams with different boundary conditions are discussed (sliding-sliding, pinned-pinned, and free-free) and, for each case, the critical loads are evaluated for increasing values of $\alpha L$.

Assuming a perfect adhesion between the foundation beam and the half-plane and adopting a 2D model with $n_{\mathrm{el}}=256$, the first three dimensionless critical loads $P_{\mathrm{cr}} / P_{\mathrm{cr}, E}$ are equal to $2.272,2.709,5.436$ for $\alpha L=5$ and turn out to be equal to $61.03,61.13,93.92$ for $\alpha L=25$. Therefore, comparison with the values reported in Table 1 shows that the frictionless contact condition yields buckling loads about $15 \%$ lower than those determined in the case of a perfect adhesion. 

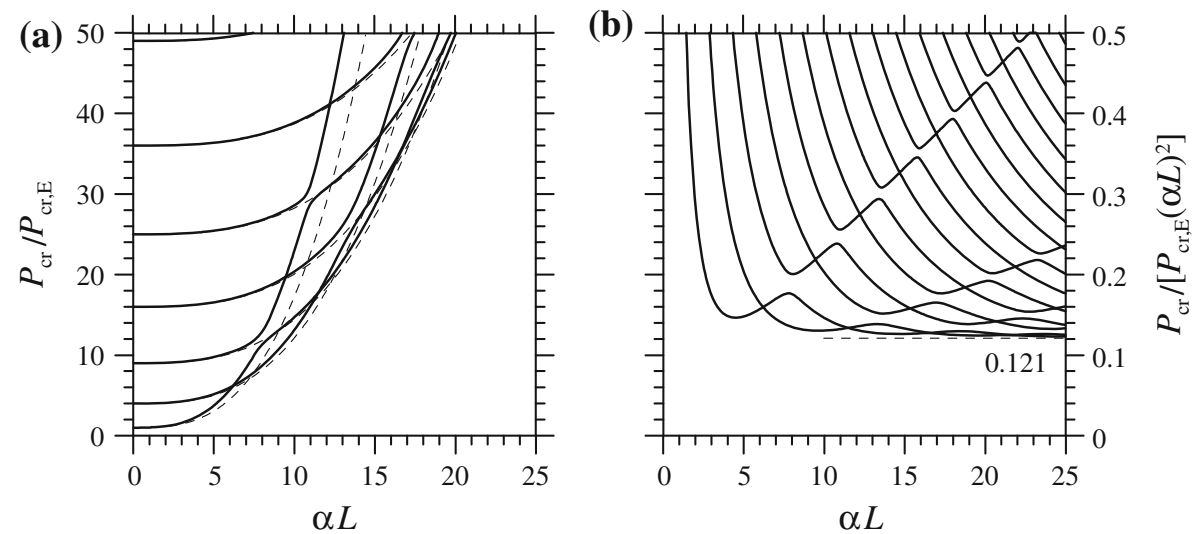

Fig. 4 Dimensionless critical loads $P_{\mathrm{cr}}$ (continuous lines) and $P_{\mathrm{cr}, m}$ (dashed lines) versus $\alpha L$ for beam with sliding ends

\subsection{Beam of finite length with sliding ends}

The case of a beam with sliding ends is considered first. This case may refer to a rectangular pipe with a top beam simply supported on rigid columns; thus, the structure prevents rotations at the ends of the foundation beam but allows independent vertical displacements. The constraint equations to be used in Eq. (4) are $R_{1}=v^{\prime}(L / 2)-v^{\prime}(-L / 2)=0$ and $R_{2}=v^{\prime}(L / 2)+v^{\prime}(-L / 2)=0$. Assuming a penalty parameter $k=10^{9} l_{i} D_{b} / L^{3}=10^{9} / 256 D_{b} / L^{2}$ that ensures a stable numerical solution of Eq. (7), the values of the first ten dimensionless buckling loads are characterized by an error lower than $10^{-5}$.

Figure $4 \mathrm{a}$ shows the first six dimensionless buckling loads $P_{\mathrm{cr}} / P_{\mathrm{cr}, E}$ versus $\alpha L$. Alternatively, Fig. $4 \mathrm{~b}$ shows the ratio $P_{\mathrm{cr}} /\left[P_{\mathrm{cr}, E}(\alpha L)^{2}\right]$. The curves in Fig. 4a, b exhibit curve veering and crossing points, and interchange themselves for increasing values of $\alpha L$, with a behavior analogous to a beam resting on Winkler soil $[11,12,18]$, where the coordinates of the intersection points may be known exactly, but the curve veering is not present.

It is worth noting that the critical loads of a beam of infinite length resting on a two-dimensional half-space and on an infinite number of equidistant supports are given by [4]:

$$
P_{\mathrm{cr}, m}=P_{\mathrm{cr}, E}\left[m^{2}+\frac{(\alpha L)^{3}}{2 m \pi^{3}}\right] \text { for } m=1,2,3, \ldots
$$

For $\alpha L=0$, that is, for a beam without supporting soil, Eq. (12) provides the buckling loads of a beam with sliding ends as well as of a simply supported beam. The first mode shape corresponds to the longest wavelength permitted by the end restraints. For $\alpha L \neq 0$, buckling loads given by Eq. (12) are close to the numerical solutions for the first two critical loads (see dashed lines in Fig. 4a). Moreover, for any given $m$, the smallest critical load $P_{\mathrm{cr}, R}$ can be obtained by substituting $\alpha L=\sqrt[3]{4} \pi m$ [4] into Eq. (12):

$$
P_{\mathrm{cr}, R}=3 m^{2} P_{\mathrm{cr}, E}=\frac{3}{\sqrt[3]{16} \pi^{2}} P_{\mathrm{cr}, E}(\alpha L)^{2}=0.121 P_{\mathrm{cr}, E}(\alpha L)^{2} .
$$

For increasing values of $\alpha L$, Fig. $4 \mathrm{~b}$ shows that the first critical load $P_{\text {cr }}$ converges to Eq. (13). In fact, for low values of $\alpha L$ (short beams and/or soft soil), the numerical solution is in good agreement with Eq. (12), whereas for high values of $\alpha L$ (long beams and/or stiff soil), it is well approximated by Eq. (13). Furthermore, Eq. (13) allows evaluation of the critical stress in a form frequently used in the design of structural sandwich panels [6-8]:

$$
\sigma_{\mathrm{cr}, R}=\frac{P_{\mathrm{cr}, R}}{b h}=\frac{3^{2 / 3}}{4} \sqrt[3]{E^{2} E_{0}}=0.52 E^{2 / 3} E_{0}^{1 / 3}
$$

For $\alpha L=5$, Fig. 5a shows the first two mode shapes that are characterized by one and two half-waves, whereas, for $\alpha L=10$, two and three half-waves are observed (Fig. 5b). As $\alpha L$ increases, short wavelengths 

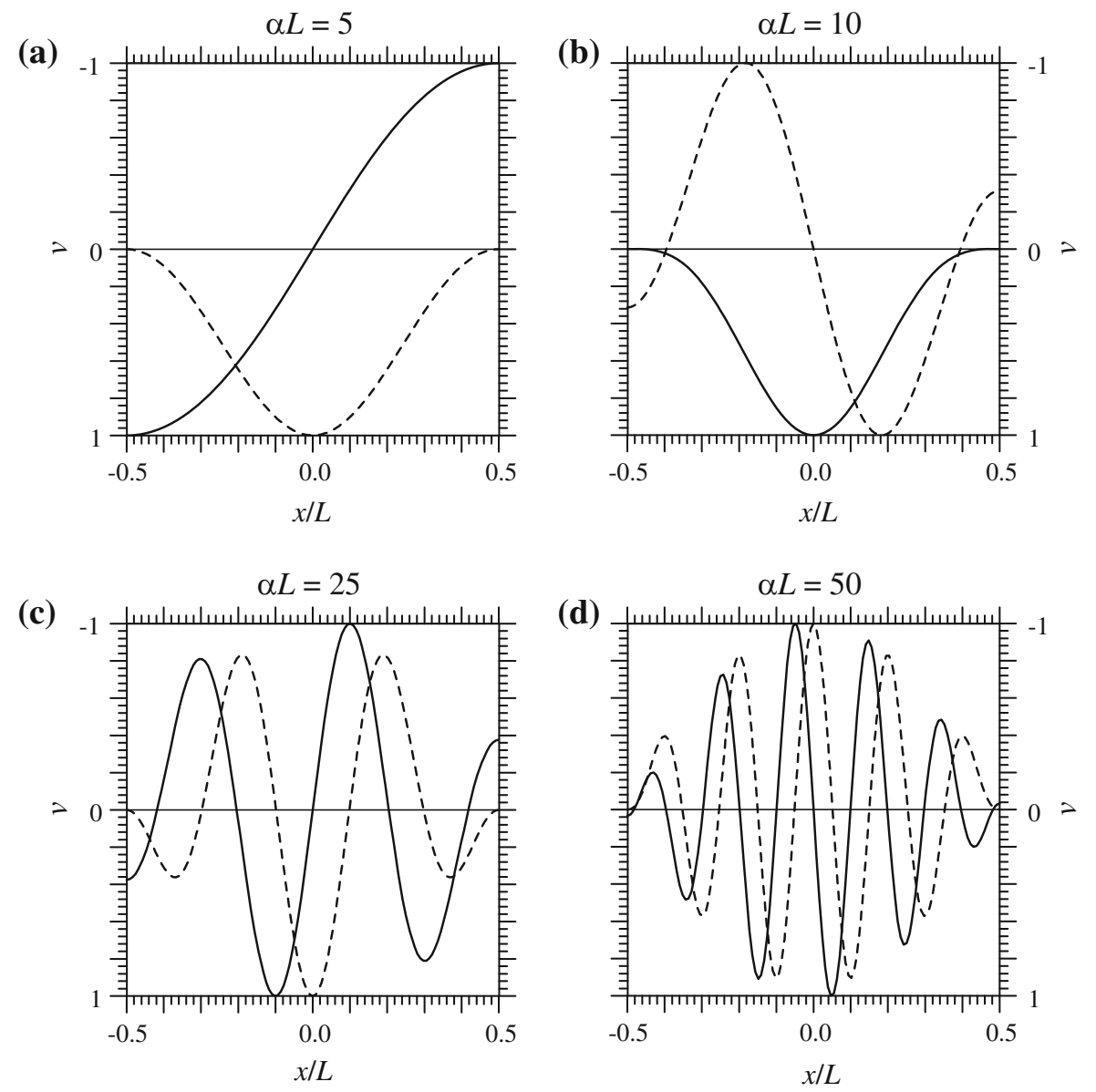

Fig. 5 First (continuous line) and second (dashed line) mode shape for a beam with sliding ends and $\alpha L$ equal to 5 (a), 10 (b), $25(\mathbf{c}), 50(\mathbf{d})$

of the first two mode shapes are obtained (Fig. 5c, d). The same behavior can be detected in beam on Winkler soil $[11,18]$. The critical wavelength $\Lambda_{\mathrm{cr}, R}$ of the sinusoidal waveform assumed in [4] is equal to [10]:

$$
\Lambda_{\mathrm{cr}, R}=2 \pi h \sqrt[3]{\frac{E_{0}}{3 E}}=\frac{2 \pi \sqrt[3]{4}}{\alpha}=\frac{9.97}{\alpha}
$$

where direct proportionality between the wavelength $\Lambda_{\mathrm{cr}, R}$ and the thickness $h$ of the beam is predicted. Eq. (15) has been used in advanced metrology methods to measure the elastic modulus of polymeric thin film [22]. Disregarding the half-waves near the beam ends, the present analysis predicts a constant critical wavelength equal to $\Lambda_{\mathrm{cr}, R}$ for at least the first two mode shapes checked. Thus, the eigenvectors shown in Fig. 5 have almost constant wavelength and variable amplitude, unlike the mode shape assumed in [4], which turns out to be sinusoidal with constant amplitude and wavelength. Usually, Euler-Bernoulli beam model holds for sufficiently high values of the critical half-wavelength, for example, $\Lambda_{\mathrm{cr}, R} / 2>10 h$; thus, Eq. (15) yields $\alpha h<1 / 2$ or equivalently $\alpha L<0.5 L / h$. For beam with $\alpha L>0.5 L / h$, the transverse shear deformation of the beam may become important and needs to be considered [23].

\subsection{Beam of finite length with pinned ends}

The case of a foundation beam with pinned ends may refer to a rigid portal frame whose columns are hinged to the foundation beam; thus, the structure enforces zero relative displacement at the beam ends, but allows independent rotations. The constraint equation to be applied to Eq. (4) is $R_{1}=v(L / 2)-v(-L / 2)=0$. 

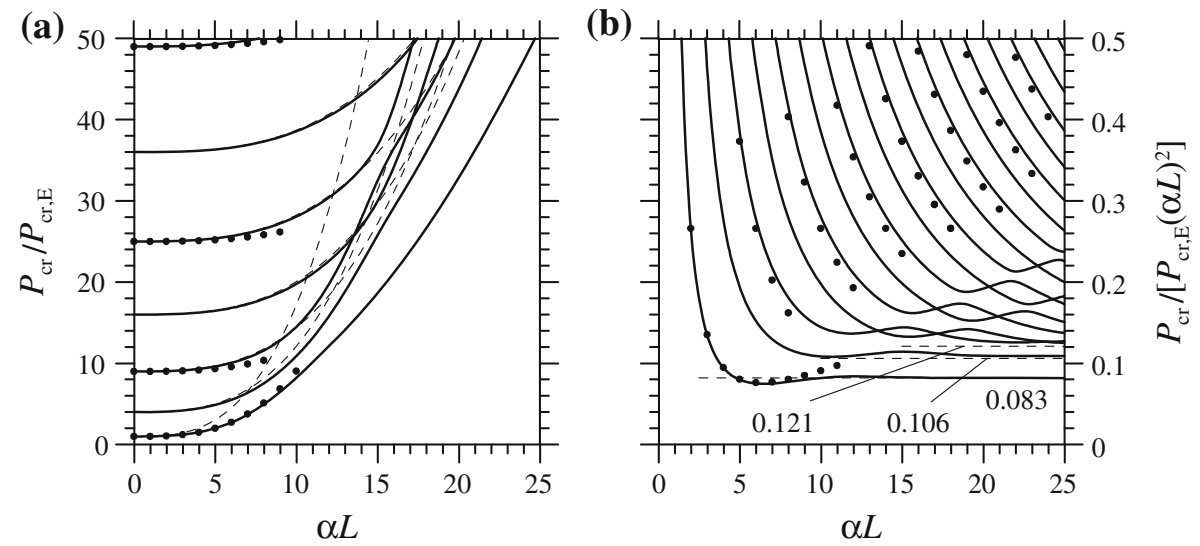

Fig. 6 Dimensionless critical loads $P_{\mathrm{cr}}$ (continuous lines), $P_{\mathrm{cr}}, m$ (dashed lines) and $P_{\mathrm{cr}, B}($ dots) versus $\alpha L$ for beam with pinned ends

Assuming a penalty parameter $k=10^{6} D_{b} / L^{3}$ that ensures a stable numerical solution of Eq. (7), the values of the first ten dimensionless buckling loads are characterized by an error lower than $10^{-5}$.

In Fig. 6a, the first seven dimensionless buckling loads $P_{\mathrm{cr}} / P_{\mathrm{cr}, E}$ are plotted versus the parameter $\alpha L$. Alternatively, Fig. 6b shows the ratio $P_{\mathrm{cr}} /\left[P_{\mathrm{cr}, E}(\alpha L)^{2}\right]$. For $\alpha L=0$, the numerical results coincide with the exact solutions given by Eq. (12) (see dashed lines in Fig. 6a). For $\alpha L \neq 0$, reference is made to the solution $P_{\mathrm{cr}, B}$ suggested in [16], where symmetric cosine functions are adopted:

$$
P_{\mathrm{cr}, B}=P_{\mathrm{cr}, E}\left[(2 m+1)^{2}+\frac{2(\alpha L)^{3}}{(2 m+1) \pi^{3}} F(m, m, 0)\right] \text { for } m=0,1,2, \ldots
$$

and the function $F(m, m, 0)$ is reported in [16]. The first buckling load is well approximated by Eq. (16) for $\alpha L<7$ (see dots in Fig. 6a, b), and by Eq. (12) for $\alpha L<3$. Moreover, Fig. 6a, b shows that Eq. (16) is unable to provide eigenvalues corresponding to antisymmetric buckling. Conversely, for increasing values of $\alpha L$, first and second critical loads converge to (Fig. 6b):

$$
\begin{aligned}
& P_{\mathrm{cr}, 1}=0.083 P_{\mathrm{cr}, E}(\alpha L)^{2}, \\
& P_{\mathrm{cr}, 2}=0.106 P_{\mathrm{cr}, E}(\alpha L)^{2},
\end{aligned}
$$

whereas third and fourth critical loads turn out to be very close to the Reissner solution and converge to Eq. (13). Therefore, the existence of critical loads lower than $P_{\mathrm{cr}, R}$ in Eq. (13) is clearly shown. In particular, Eqs. (17) and (18) yield the following critical stresses:

$$
\begin{aligned}
& \sigma_{\mathrm{cr}, 1}=\frac{P_{\mathrm{cr}, 1}}{b h}=0.36 E^{2 / 3} E_{0}^{1 / 3}, \\
& \sigma_{\mathrm{cr}, 2}=\frac{P_{\mathrm{cr}, 2}}{b h}=0.46 E^{2 / 3} E_{0}^{1 / 3} .
\end{aligned}
$$

For $\alpha L=5$, Fig. 7a shows that first and second mode shapes present one and two half-waves, respectively, whereas for $\alpha L=25$ (Fig. 7b), the half-waves cannot be easily defined like in previous cases and the buckling modes show the greatest amplitudes near the beam ends. Indeed, the critical loads in Eqs. (17) and (18) correspond to these localized buckling modes.

The behavior of the beam with pinned ends on an elastic half-space is found to be different from that of the beam with sliding ends, which does not present localized eigenmodes for increasing values of $\alpha L$ (long beam and/or stiff soil). With regard to Winkler soil, reference is usually made to [12], where the solution of the beam with pinned ends converges to the critical load of the beam with clamped ends [12,24]. Nonetheless, for a beam with pinned ends resting on Winkler soil, Goodier and Hsu [13] have shown the occurrence of mode shapes localized at the beam ends. 

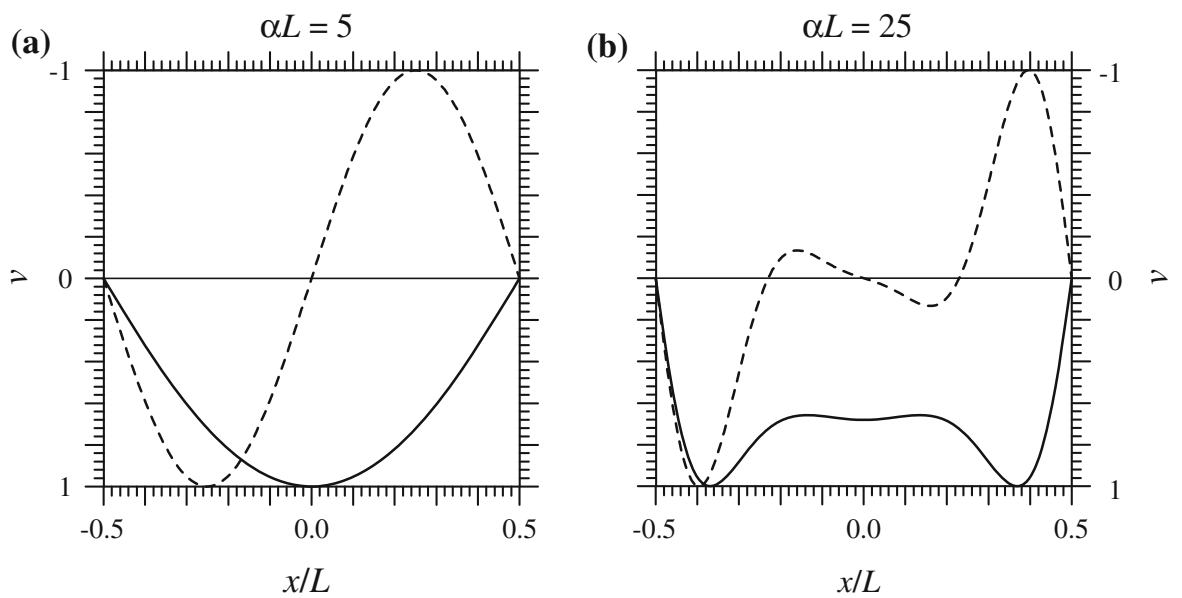

Fig. 7 First (continuous line) and second (dashed line) mode shape for a beam with pinned ends and $\alpha L$ equal to 5 (a), 25 (b)
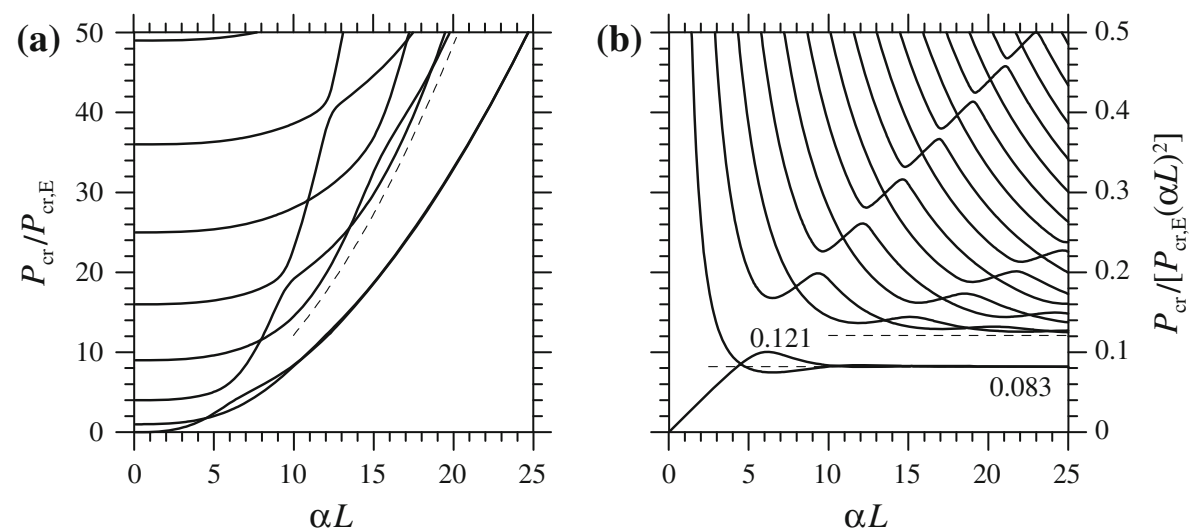

Fig. 8 Dimensionless critical loads $P_{\mathrm{cr}}$ (continuous lines) and $P_{\mathrm{cr}, R}$ (dashed line) versus $\alpha L$ for beam with free ends

\subsection{Beam of finite length with free ends}

In Fig. 8a, the dimensionless critical loads $P_{\mathrm{cr}} / P_{\mathrm{cr}, E}$ are plotted versus $\alpha L$ values; the dashed line shows the function given by Eq. (13). However, the first and second eigenvalues appear quite far from this solution and, for increasing $\alpha L$, converge to the critical load $P_{\mathrm{cr}, 1}$ in Eq. (17). Indeed, it is worth noting that a beam with free ends and symmetric behavior is practically coincident with a beam with pinned ends. It is interesting to observe that the first and second eigenvalues are quite different for very small values of $\alpha L$ and present some intersection points, whereas for $\alpha L \geq 10$, they both converge to the value given by Eq. (17). For increasing values of $\alpha L$, the third and fourth eigenvalues are close to Reissner solution and converge to the value given by Eq. (13) for the beam with sliding ends.

In absence of soil, the critical value of a beam with free ends is obviously zero, but for very short beam or very soft soil $(\alpha L=1)$, the first mode is antisymmetric and represents a rigid body movement, whereas the second one is symmetric and sinusoidal with one half-wave (Fig. 9a). For $\alpha L=5$, the first mode shape becomes symmetric and the second one antisymmetric (Fig. 9b). Moreover, for $\alpha L \geq 5$, the first and second critical loads are very close to the one given by Eq. (17) and both mode shapes tend to be localized near the beam ends, as shown for $\alpha L=25$ in Fig. 9c, where the first mode shape is coincident with the one found for the beam with pinned ends (Fig. 7b).

Figure 10 shows the first mode shape for a beam with sliding ends, and the third mode shape for beams with pinned and free ends resting on a stiff soil $(\alpha L=25)$. Eigenvectors are practically coincident in the neighborhood of beam midpoint and present small differences at the beam ends, due to the different restraints. 


$$
\alpha L=1
$$

(a)

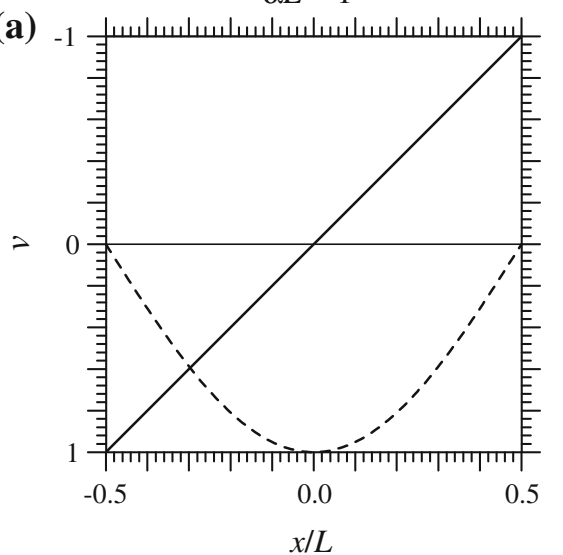

$\alpha L=25$

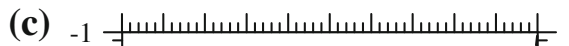

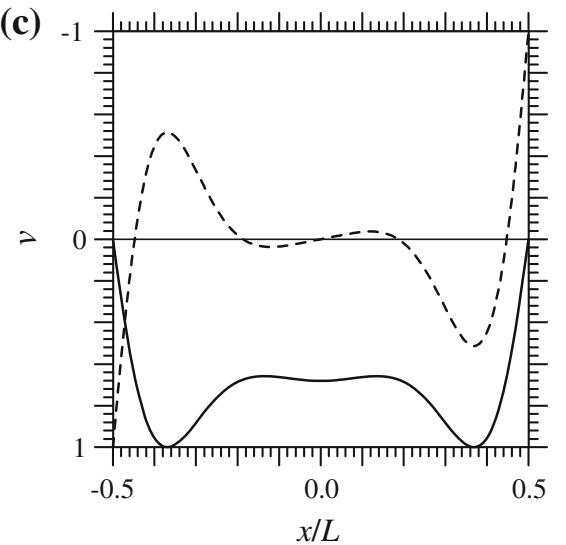

$\alpha L=5$

(b)

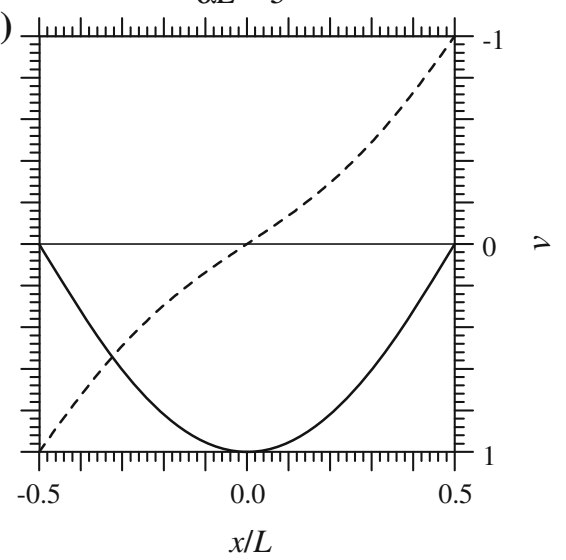

$\alpha L=50$

(d)

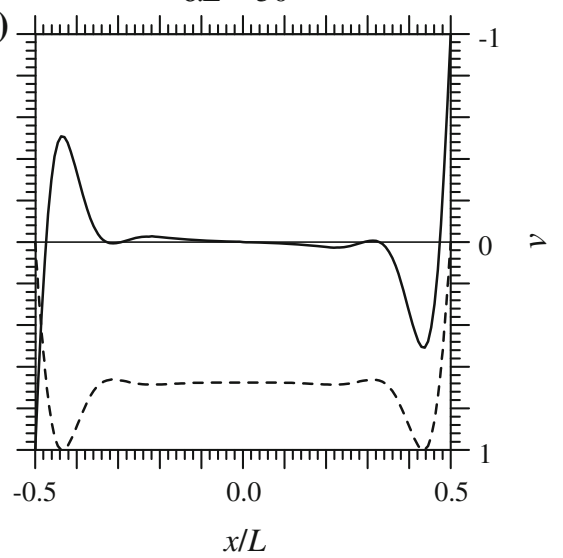

Fig. 9 First (continuous line) and second (dashed line) mode shape for a beam with free ends and $\alpha L$ equal to 1 (a), 5 (b), 25 (c), 50 (d)

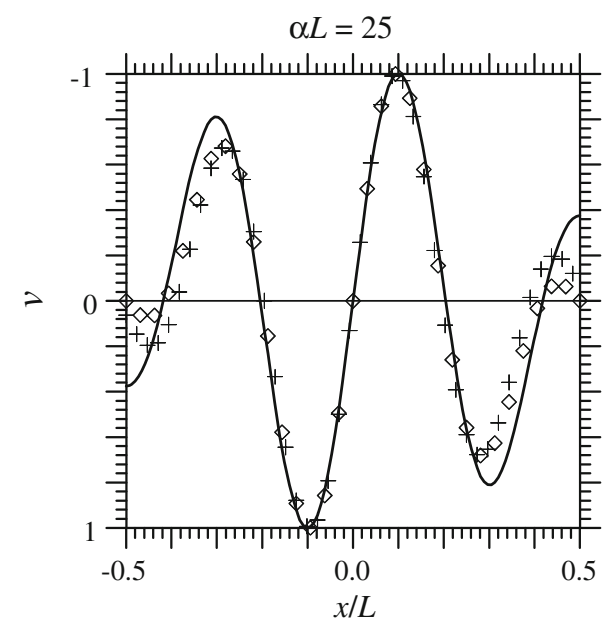

Fig. 10 First mode shape for a beam with sliding ends (continuous line), and third mode shape for beam with pinned (diamond symbol) and free (plus symbol) ends resting on stiff soil $(\alpha L=25)$

The behavior of the beam with free ends on an elastic half-space is quite similar to the corresponding case of beam on Winkler soil, where first and second critical loads converge to one half of the smallest critical load of a beam with clamped or sliding ends $[12,18,24]$. 
Table 2 First four dimensionless critical loads $P_{\mathrm{cr}} /\left[P_{\mathrm{cr}, E}(\alpha L)^{2}\right]$ for beams of infinite length with different boundary conditions

\begin{tabular}{lllll}
\hline End restraints & 1st & 2nd & 3rd & 4th \\
\hline Free-free & 0.083 & 0.083 & 0.121 & 0.121 \\
Pinned-pinned & 0.083 & 0.106 & 0.121 & 0.121 \\
Sliding-free & 0.083 & 0.121 & 0.121 & 0.125 \\
Sliding-pinned & 0.094 & 0.121 & 0.121 & 0.125 \\
Sliding-sliding & 0.121 & 0.121 & 0.125 & 0.125 \\
\hline
\end{tabular}

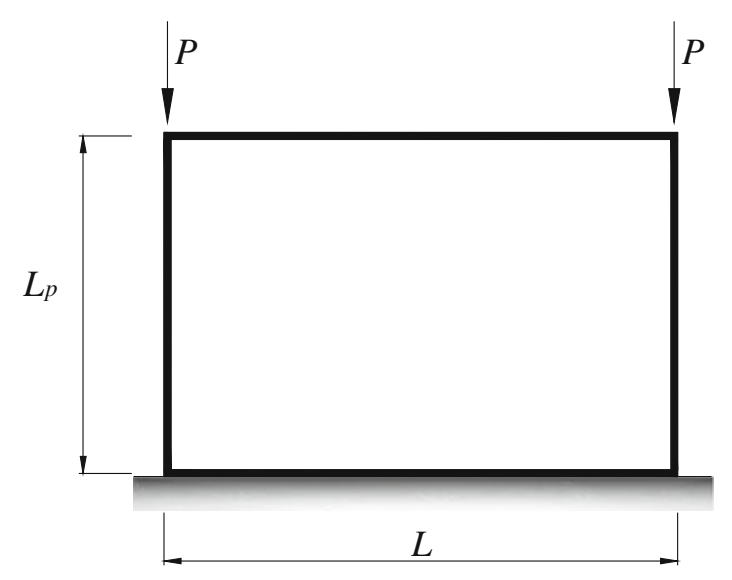

Fig. 11 Rectangular pipe with compressed columns

\subsection{Beam of infinite length with different boundary conditions}

Figures $4 \mathrm{~b}, 6 \mathrm{~b}, 8 \mathrm{~b}$ show that the dimensionless critical loads converge to well definite limits; consequently, sufficiently high value of $\alpha L$ allows to consider beams as if they were of infinite length. Table 2 collects the first four dimensionless critical loads $P_{\mathrm{cr}} /\left[P_{\mathrm{cr}, E}(\alpha L)^{2}\right]$ for beam with $\alpha L=50$ and different boundary conditions. In particular, in the sliding-pinned case, the constraint equations that have to be used in Eq. (4) are $R_{1}=v(L / 2)-v(-L / 2)=0$ and $R_{2}=v^{\prime}(-L / 2)-[v(-L / 2)-v(L / 2)] / L=0$, whereas in the sliding-free case, the constraint equation is $R_{1}=v^{\prime}(-L / 2)=0$. In this case, the first buckling mode is localized at the free end. Reissner result given by Eq. (13) is well achieved by beams with two sliding ends. Nevertheless, beams with different boundary conditions show that the first critical load is significantly smaller than that obtained by Reissner solution.

\section{Rectangular pipe on an elastic half-plane}

As suggested in the static case [17], the proposed model allows us to study easily the coupling of the foundation beam with structures described by traditional FEs. To this end, a rectangular frame of length $L$ and height $L_{p}$ is considered (Fig. 11); both the foundation and the top beam have the same bending rigidity $D_{b}$, whereas $D_{p}$ indicates the column bending rigidity. Concentrated forces $P$ are applied at upper column ends. In the following, introducing the dimensionless parameters

$$
\eta=\frac{L}{L_{p}}, \quad \rho=\frac{D_{b}}{D_{p}}, \quad \psi=\frac{\rho}{\eta}, \quad \lambda_{p}=\frac{P L_{p}^{2}}{D_{p}}=\frac{\rho}{\eta^{2}} \lambda,
$$

two cases with $\psi=0.5(\rho=1, \eta=2)$ and $\psi=2.0(\rho=4, \eta=2)$ are considered. A number of 32 equal FEs is adopted for the foundation beam, whereas the other frame elements are subdivided into 16 equal FEs.

\subsection{Buckling of rectangular pipes with free and pinned foundation beam ends}

Buckling analyses are restricted to the determination of the first two critical loads and the corresponding mode shapes. Free and pinned foundation beam ends are considered, and in the latter case, the penalty function $R_{1}$ introduced in Sect. 3.3 is used. 

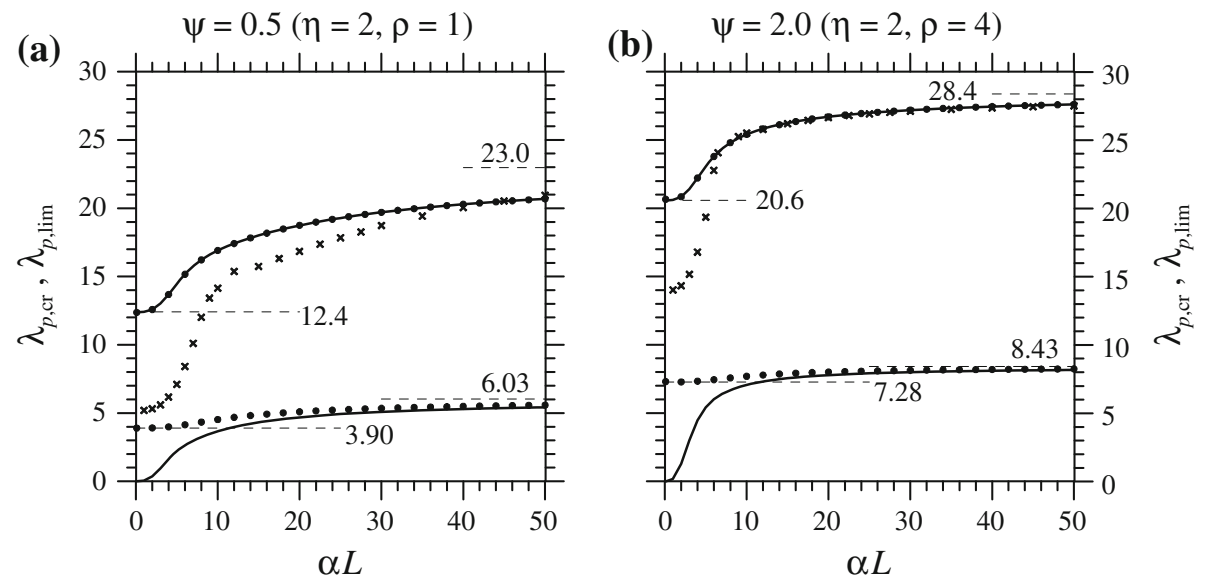

Fig. 12 Rectangular pipe with $\psi=0.5$ (a) and 2.0 (b). First and second dimensionless critical loads $\lambda_{p \text {,cr }}$ (continuous lines and $d o t s$ ) and $\lambda_{p, \lim }$ (cross symbol) versus $\alpha$ L. Continuous lines and dots describe pipe with free and pinned foundation ends, respectively

Figure 12a, b shows the first two dimensionless critical loads $\lambda_{p, \text { cr }}$ as a function of $\alpha L$ for $\psi=0.5$ and $\psi=2.0$, respectively. Continuous lines represent the pipe with free foundation ends, whereas dots represent critical loads of the frames with pinned foundation ends. For very soft soil and free foundation ends, the first critical load tends to zero and the corresponding mode shape is a rigid body movement (Fig. 13a), whereas, for pipe with pinned foundation ends (Fig. 13c), the first critical load is close to the analytical solution of the rectangular pipe without supporting soil, that is, $\lambda_{p, \text { cr }}=3.90$ for $\psi=0.5$ and $\lambda_{p \text {,cr }}=7.28$ for $\psi=2.0$. For very stiff soil and both restraint conditions, foundation deflections are small (Fig. 13b, d) and the first critical load converges to the critical load of a portal frame with built-in bases $\left(\lambda_{p, \text { cr }}=6.03\right.$ for $\psi=0.5$ and $\lambda_{p, \mathrm{cr}}=8.43$ for $\psi=2.0$ ).

With regard to the second eigenvalue, both restraint conditions give equal buckling loads and identical mode shapes (Fig. 13e, f); thus, a pipe with free ends behaves like one with pinned foundation ends. For very soft substrate, soil reactions extend along the whole foundation length. Therefore, the second critical load can be overestimated by the corresponding value $\lambda_{p \text {,cr,sup }}$ of a pipe without supporting soil and it can be underestimated by the critical load $\lambda_{p, \text { cr,inf }}$ of a self-equilibrate pipe having concentrated forces at top column ends and a distributed load equal to $2 P / L$ on the foundation beam, which simulates constant soil reactions. For $\psi=0.5$ and $\alpha L$ tending to zero, the second critical load tends to $\lambda_{p, c r}=12.4$ (Fig. 12a), bounded by $\lambda_{p, \text { cr,inf }}=11.6$ and $\lambda_{p, \text { cr,sup }}=13.5$. Moreover, the symmetric mode shape depicted in Fig. 13e clearly shows a foundation deflection larger than the upper beam deflection. This behavior is caused by the compressive force in foundation beam, which reduces its flexural stiffness. For $\psi=2.0$ and $\alpha L$ tending to zero, the second critical load tends to $\lambda_{p, \text { cr }}=20.6$ (Fig. 12 b), bounded by $\lambda_{p \text {,cr,inf }}=20.3$ and $\lambda_{p \text {,cr,sup }}=21.6$. For increasing $\alpha L$, the second critical load converges to the corresponding value of a portal frame with built-in bases $\left(\lambda_{p, \text { cr }}=23.0\right.$ for $\psi=0.5, \lambda_{p, \text { cr }}=28.4$ for $\left.\psi=2.0\right)$. For pipes having the same geometry, but different bending rigidity ratios $\rho$, the stiffer the foundation beam, the lower the soil stiffness necessary to converge to a portal frame with built-in bases. Hence, for $\psi=2.0(\rho=4, \eta=2)$, Fig. 12b shows that convergence to $\lambda_{p, \text { cr }}=28.4$ is almost achieved for $\alpha L>50$, whereas, for $\psi=0.5(\rho=1, \eta=2)$, Fig. 12a shows that convergence to $\lambda_{p, \mathrm{cr}}=23.0$ is slower and the mode shape depicted in Fig. $13 \mathrm{f}$ is characterized by small foundation deflections.

\subsection{Nonlinear incremental analyses of rectangular pipes}

With regard to the second mode shape, the axial forces in the columns are statically determinate and are equal to $P$, but the axial forces in top and foundation beams are statically indeterminate and depend nonlinearly on the load multiplier $\lambda_{p}$; thus, a snap-through instability is to be expected [18]. In order to obtain a more correct study of the pipe stability, nonlinear incremental analyses are carried out, taking into account the axial force variation in top and foundation beams. To this purpose, the axial degrees of freedom are added to the beam FEs and the element stiffness matrix is modified as usual [20]; the axial-to-bending rigidity ratio $E_{0} b h / D_{b}$ is 
(a)

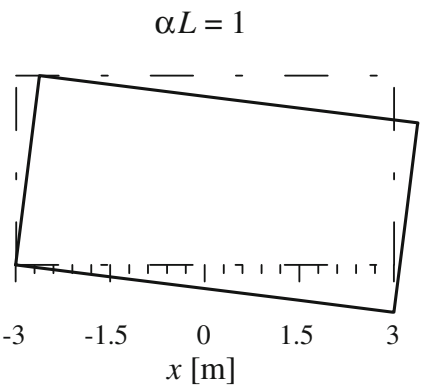

(c)

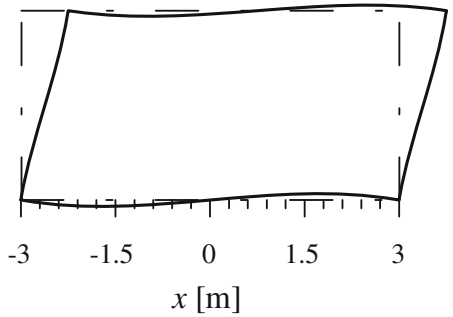

(e)

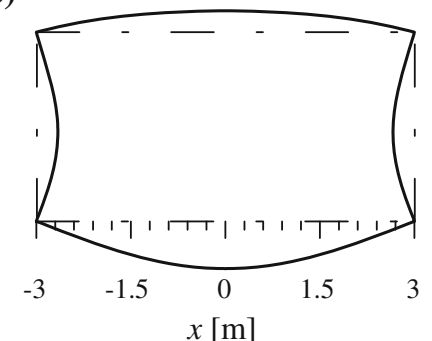

(b)

$\alpha L=25$

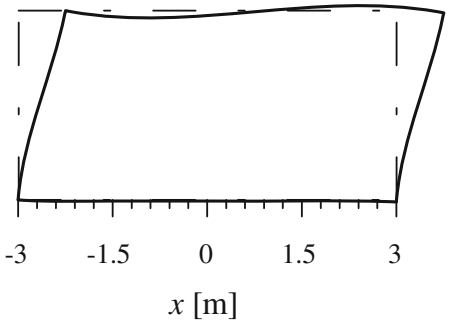

(d)

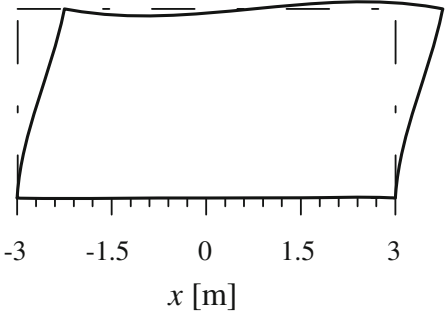

(f)

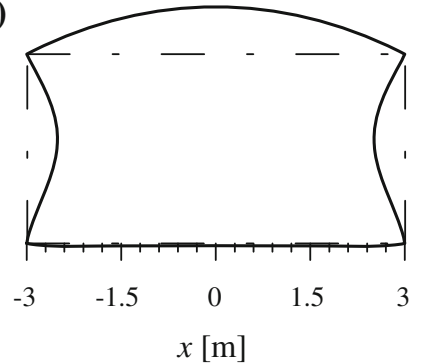

Fig. 13 Rectangular pipe with $\psi=0.5$. Mode shapes for $\alpha L=1(\mathbf{a}, \mathbf{c}, \mathbf{e})$, and $25(\mathbf{b}, \mathbf{d}, \mathbf{f})$. First mode shape for a pipe with free $(\mathbf{a}, \mathbf{b})$ and pinned $(\mathbf{c}, \mathbf{d})$ foundation ends. Second mode shape $(\mathbf{e}, \mathbf{f})$ for a pipe with either free or pinned foundation ends

taken equal to $12 / h^{2}$ with beam slenderness $L / h=10$. Displacement control has been performed by using the formulation proposed in [25].

With regard to the pipe with $\rho=1, \eta=2(\psi=0.5)$ and $\alpha L=1$, Fig. 14a shows load multiplier $\lambda_{p}$ as a function of the rotation $\phi$ at the foundation beam end. In particular, the straight line No. 1 represents the linear analysis, the curve No. 2 corresponds to a nonlinear incremental analysis that takes into account second-order effects, but assumes axial forces proportional to those obtained with the linear analysis; in this case, load multiplier $\lambda_{p}$ converges to $\lambda_{p, \text { cr }}=12.4$, determined with the buckling analysis. The curve No. 3 represents a nonlinear incremental analysis that upgrades the axial forces in each load step and the load multiplier $\lambda_{p}$ attains the limit point $\lambda_{p, \lim }=5.2$, which is $58 \%$ lower than $\lambda_{p, \mathrm{cr}}=12.4$ determined with the buckling analysis. Figure 14b shows the load multiplier $\lambda_{p}$ as a function of the dimensionless axial force in the foundation beam $N_{b} L_{p}^{2} / D_{p}$ (positive sign is assigned to compression forces), and $N_{b}$ increases more than linearly with respect to the load multiplier $\lambda_{p}$, up to the attainment of the buckling load of the foundation beam.

For other values of $\alpha L$, the load multipliers $\lambda_{p, \text { lim }}$ at limit point are determined and added to Fig. 12a by using cross symbols. The differences between buckling and nonlinear incremental analysis are large up to $\alpha L=10$, whereas for stiffer soil, the load multipliers $\lambda_{p, \lim }$ are quite close to the buckling loads $\lambda_{p, \mathrm{cr}}$. For pipe with $\rho=4, \eta=2(\psi=2.0)$, Fig. 12b shows that differences between buckling and nonlinear incremental analysis are large up to $\alpha L=5$.

Finally, for $\alpha L=1$ and 5, Tables 3 and 4 show the ratio $\lambda_{p, \lim } / \lambda_{p, \text { cr }}$ for some values of the pipe parameters $\rho$ and $\eta$. Each diagonal in Tables 3 and 4 exhibits the same value of $\psi$, which provides the same $\lambda_{p \text {,cr }}$ regardless of the chosen parameters $\rho$ and $\eta$. Conversely, small limit load multiplier $\lambda_{p}$,lim occurs with low values of $\rho$ and if $L>2 L_{p}$. However, Fig. 14a shows that the limit load multiplier $\lambda_{p, \text { lim }}$ requires significant rotation. Consequently, ordinary structures collapse for the achievement of strength limit rather than by elastic instability. 

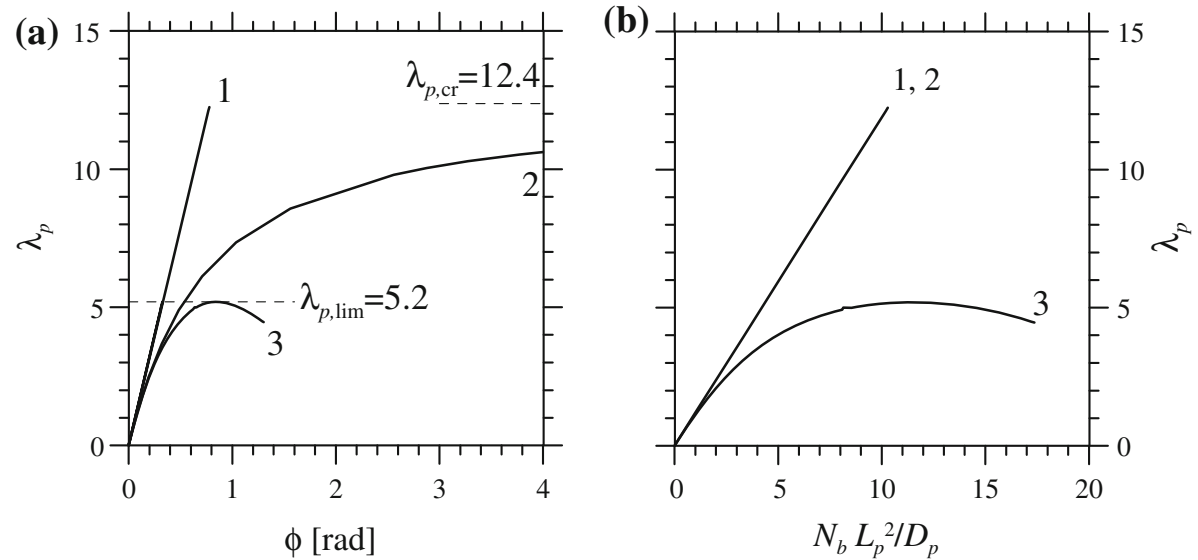

Fig. 14 Rectangular pipe with $\psi=0.5$ and $\alpha L=1$. Load multiplier $\lambda_{p}$ as a function of the rotation $\phi$ at the foundation beam end (a) and the dimensionless axial force in foundation beam $N_{b} L_{p}^{2} / D_{p}$ (b) for linear analysis (straight line 1), nonlinear incremental analysis with second-order effects (curve 2), nonlinear incremental analysis that upgrade the axial forces in each load step (curve 3)

Table 3 Ratio $\lambda_{p, \lim } / \lambda_{p, \text { cr }}$ for rectangular pipes on an elastic half-plane with $\alpha L=1$

\begin{tabular}{lllll}
\hline$\alpha L=1$ & $\eta=L / L_{p}$ & & \\
\cline { 2 - 5 } & 0.5 & 1 & 2 & 4 \\
\hline$\rho=D_{b} / D_{p}$ & 1.00 & 0.80 & 0.52 & 0.42 \\
0.5 & 1.00 & 1.00 & 0.53 & 0.27 \\
1 & 1.00 & 0.99 & 0.68 & 0.29 \\
4 & 1.00 & 1.00 & & 0.32 \\
\hline
\end{tabular}

Table 4 Ratio $\lambda_{p, \lim } / \lambda_{p, \text { cr }}$ for rectangular pipes on an elastic half-plane with $\alpha L=5$

\begin{tabular}{lllll}
\hline$\alpha L=5$ & $\eta=L / L_{p}$ & & \\
\cline { 2 - 5 } & 0.5 & 1 & 2 & 4 \\
\hline$\rho=D_{b} / D_{p}$ & 1.00 & 0.96 & 0.65 & 0.49 \\
0.5 & 1.00 & 1.00 & 0.65 & 0.25 \\
1 & 1.00 & 0.96 & 0.84 & 0.26 \\
2 & 1.00 & 1.00 & & 0.31 \\
\hline
\end{tabular}

\section{Conclusions}

A simple and effective FE-BIE coupling method is proposed and applied to stability problems of beams on an elastic half-plane having finite length and different end restraints. The proposed coupled FE-BIE model has turned out to be fast and effective in evaluating buckling loads and corresponding mode shapes. The adopted Euler-Bernoulli beam model holds if $\alpha L<0.5 L / h$; conversely, the transverse shear deformation of the beam may become important and needs to be considered [23]. For increasing beam slenderness and/or soil stiffness, a variation of the critical loads proportional to $(\alpha L)^{2}$ has been found. For low values of $\alpha L$, the well-known analytic results derived by Reissner [4] for a beam of infinite length on an elastic half-plane are quite similar to those of a beam with either pinned or sliding ends, whereas, for large values of $\alpha L$, Reissner results are well achieved by beams with sliding ends. Nevertheless, beams with pinned or free ends show that the first two critical loads are significantly smaller than those obtained by Reissner solution. The corresponding mode shapes are characterized by large amplitudes in the neighborhood of the beam ends, whereas third and fourth critical loads converge to the value given by Reissner. Finally, nonlinear incremental analyses of rectangular frames with compressed columns and free or pinned foundation ends have been considered. As for pipes with pinned foundation ends, snap-through instability is significant for pipes stiffer than the soil.

Mixed variational principle, similar to the one presented in [17], was used in [26] to study an axially loaded thin structures perfectly bonded to an elastic substrate and in [23] to determine the buckling loads of Timo- 
shenko beams in frictionless contact with an elastic half-plane. Future investigations might adopt alternative Green functions, for example, associated with an elastic non-homogeneous half-plane or with a finite thickness layer resting on a rigid base. The generalization of the proposed method to contact problems with a poroelastic substrate or friction requires more detailed investigations.

Acknowledgments The present investigation was developed in the framework of the two Italian Research Programs No. 2009XWLFKW (coordinated by Prof. Alberto Corigliano from the Polytechnic of Milan) and No. 20089RJKYN (coordinated by Prof. Franco Maceri of the University of Rome "Tor Vergata"), the Research Program FAR 2011 of the University of Ferrara, and the Research Program funded by the Italian Civil Protection National Service - Executive Project 2010-2013 within the activities of the (Italian) University Network of Seismic Engineering Laboratories-ReLUIS. The financial support of the Italian Ministry for University and Research, the University of Ferrara and ReLUIS is gratefully acknowledged.

\section{Appendix}

For a prismatic beam element subjected to uniform loads $p(x)$ and with constant soil pressure, element matrices appearing in Eq. (5) are

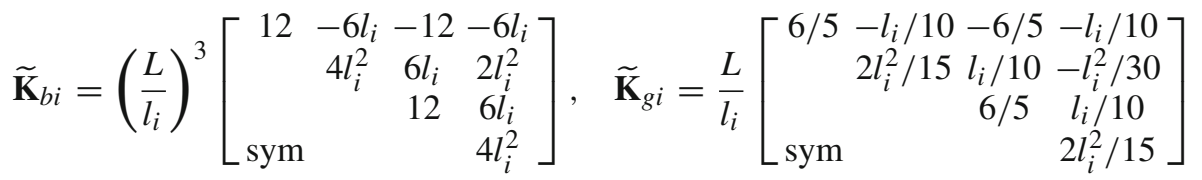

$$
\begin{aligned}
& \mathbf{F}_{i}=p\left[l_{i} / 2,-l_{i}^{2} / 12, l_{i} / 2, l_{i}^{2} / 12\right]^{\mathrm{T}}, \quad \mathbf{H}_{i}=\left[l_{i} / 2,-l_{i}^{2} / 12, l_{i} / 2, l_{i}^{2} / 12\right]^{\mathrm{T}}, \\
& \widetilde{g}_{i i}=\frac{2}{\pi} l_{i}^{2}\left(\frac{3}{2}-\ln l_{i}\right) \text {, } \\
& \tilde{g}_{i j}=\frac{2}{\pi}\left[\frac{3}{2} l_{i} l_{j}+G\left(x_{j+1}-x_{i+1}\right)-G\left(x_{j+1}-x_{i}\right)-G\left(x_{j}-x_{i+1}\right)+G\left(x_{j}-x_{i}\right)\right] \quad \text { for } i \neq j
\end{aligned}
$$

where $G(x)=x^{2} / 2 \ln |x|$.

\section{References}

1. Wieghardt, K.: Über den Balken auf nachgiebiger Unterlage. ZAMM-Zeitschrift für Angewandte Mathematik Und Mechanik 2(3), 165-184 (1922)

2. Prager, W.: Zur Theorie elastische gelagerter Konstruktionen. ZAMM-Zeitschrift für Angewandte Mathematik Und Mechanik 7(5), 354-360 (1927)

3. Biot, M.A.: Bending of an infinite beam on an elastic foundation. J. Appl. Mech. Trans. ASME 4, A1-A7 (1937)

4. Reissner, M.E.: On the theory of beams resting on a yielding foundation. Proc. Natl. Acad. Sci. U.S.A. 23(6), 328-333 (1937)

5. Gough, G.S., Elam, C.F., de Bruyne, N.A.: The stabilisation of a thin sheet by a continuous supporting medium. J. R. Aeronaut. Soc. 44, 12-43 (1940)

6. Allen, H.G.: Analysis and Design of Structural Sandwich Panels. Pergamon Press, Oxford (1969)

7. Ley, R.P., Lin, W., Mbanefo, U.: Facesheet wrinkling in sandwich structures. CR-1999-208994, NASA, Virginia (1999)

8. Davies, J.M.: Lightweight Sandwich Construction. Blackwell Science, Oxford (2001)

9. Shield, T.W., Kim, K.S., Shield, R.T.: The buckling of an elastic layer bonded to an elastic substrate in plane strain. J. Appl. Mech. TranS. ASME 61, 231-235 (1994)

10. Volynskii, A.L., Bazhenov, S., Lebedeva, O.V., Bakeev, N.F.: Mechanical buckling instability of thin coatings deposited on soft polymer substrates. J. Mater. Sci. 35, 547-554 (2000)

11. Timoshenko, S.P., Gere, J.M.: Theory of Elastic Stability. McGraw-Hill, New York (1961)

12. Hetenyi, M.: Beam on Elastic Foundation. The University of Michigan Press, Ann Arbor (1946)

13. Goodier, J.N., Hsu, C.S.: Nonsinusoidal buckling modes of sandwich plates. J. Aeronaut. Sci. 21, 525-532 (1954)

14. Smith, T.E.: Buckling of a beam on a Wieghardt-type elastic foundation. ZAMM-Zeitschrift für Angewandte Mathematik Und Mechanik 49(11), 641-645 (1969)

15. Gallagher, A.P: Buckling of a beam under axial compression with elastic support. In: Scaife, B.K.P. (ed.) Studies in Numerical Analysis, pp. 137-150. Academic Press, London (1974)

16. Bosakov, S.V.: Variational approach to the solution of a contact problem for an elastic half-plane. Int. Appl. Mech. 30(7), 535-538 (1994)

17. Tullini, N., Tralli, A.: Static analysis of Timoshenko beam resting on elastic half-plane based on coupling of locking-free elements and boundary integral. Comput. Mech. 45(2-3), 211-225 (2010)

18. Bazant, Z.P., Cedolin, L.: Stability of Structures. Oxford University Press, New York (1991)

19. Johnson, K.L.: Contact Mechanics. Cambridge University Press, Cambridge (1985) 
20. Reddy, J.N.: An Introduction to the Finite Element Method, 3rd edn. McGraw Hill, Singapore (2006)

21. Vesic, A.B.: Bending of beams resting on isotropic elastic solid. J. Eng. Mech. Div. ASCE 87(EM2), 35-53 (1961)

22. Stafford, C.M., Harrison, C., Beers, K.L., Karim, A., Amis, E.J., VanLandingham, M.R., Kim, H.-C., Volksen, W., Miller, R.D., Simonyi, E.E.: A buckling-based metrology for measuring the elastic moduli of polymeric thin films. Nat. Mater. 3(8), 545-550 (2004)

23. Tullini, N., Tralli, A., Baraldi, D.: Buckling of Timoshenko beams in frictionless contact with an elastic half-plane. J. Eng. Mech. ASCE. doi:10.1061/(ASCE)EM.1943-7889.0000529

24. Simitses, G.J.: An Introduction to Elastic Stability of Structures. Prentice-Hall, Englewood Cliffs (1976)

25. Batoz, J.L., Dhatt, G.: Incremental displacement algorithms for non-linear problems. Int. J. Numer. Methods Eng. 14(8), 1262-1267 (1979)

26. Tullini, N., Tralli, A., Lanzoni, L.: Interfacial shear stress analysis of bar and thin film bonded to 2D elastic substrate using a coupled FE-BIE method. Finite Elem. Anal. Des. 55, 42-51 (2012) 